\title{
Training-induced changes in subsequent-memory effects: No major differences among children, younger adults, and older adults
}

\author{
Yvonne Brehmer ${ }^{\mathrm{a}, \mathrm{b}, *}$, Yee Lee Shing ${ }^{\mathrm{b}, \mathrm{c}}$, Hauke R. Heekeren ${ }^{\mathrm{d}}$, Ulman Lindenberger ${ }^{\mathrm{b}, \mathrm{e}}$, Lars Bäckman ${ }^{\mathrm{a}}$ \\ a Aging Research Center, Karolinska Institutet and Stockholm University, Stockholm, Sweden \\ b Center for Lifespan Psychology, Max Planck Institute for Human Development, Berlin, Germany \\ c School of Natural Sciences, University of Stirling, Stirling, UK \\ d Department of Education Sciences and Psychology, Freie Universität, Berlin, Berlin, Germany \\ e Department of Political and Social Sciences, European University Institute, San Domenico di Fiesole, Fiesole, Italy
}

\section{A R T I C L E I N F O}

\section{Article history:}

Accepted 30 November 2015

Available online 7 December 2015

\section{Keywords:}

Episodic memory

Training

Subsequent-memory effect

Functional magnetic resonance imaging

Lifespan

\begin{abstract}
A B S T R A C T
The neural correlates of encoding mode, or the state of forming new memory episodes, have been found to change with age and mnemonic training. However, it is unclear whether neural correlates of encoding success, termed subsequent-memory (SM) effects, also differ by age and mnemonic skill. In a multi-session training study, we investigated whether SM effects are altered by instruction and training in a mnemonic skill, and whether such alterations differ among children, younger adults, and older adults. Before and after strategy training, fMRI data were collected while participants were memorizing word pairs. In all age groups, participants receiving training showed greater performance gains than control group participants. Analysis of task-relevant regions showed training-induced reductions in SM effects in left frontal regions. Reductions in SM effects largely generalized across age and primarily reflected greater training-induced activation increases for omissions than for remembered items, indicating that training resulted in more consistent use of the mnemonic strategy. The present results reveal no major age differences in SM effects in children, younger adults, and older adults.
\end{abstract}

(c) 2015 Elsevier Inc. All rights reserved.
Episodic memory (EM; Tulving, 1972), the ability to remember events situated in time and place, increases from childhood to early adulthood (Schneider and Pressley, 1997) and decreases from around age 60 and onwards (Kausler, 1994; Rönnlund et al., 2005). Several studies have investigated if the generally lower EM performance of older adults can be increased through strategy-based training. These studies typically demonstrate that older adults benefit from strategy training, either to a lesser (Ball et al., 2002; Kliegl and Baltes, 1987; Kliegl et al., 1990; Singer et al., 2003) or similar (Bherer et al., 2006; Derwinger et al., 2003; Nyberg et al., 2003) extent compared to younger adults. In previous work, we found that children performed similarly to older adults before training or after being instructed in a mnemonic strategy, although they profited more than older adults from subsequent adaptive practice in the strategy (Brehmer et al., 2007; Shing et al., 2008).

Regarding neural correlates of training-induced changes in memory across the lifespan, not much work has been conducted so far. To our knowledge, no study has investigated training-induced neural correlates of changes in EM functioning during childhood. Research examining training-induced activation changes in adulthood reveals activity changes following instruction and practice in mnemonic techniques or

\footnotetext{
* Corresponding author at: Aging Research Center, Gävlegatan 16, 11330 Stockholm, Sweden.

E-mail address: yvonne.brehmer@ki.se (Y. Brehmer).
}

semantic encoding strategies (Jones et al., 2006; Kirchhoff et al., 2012; Nyberg et al., 2003), mostly in prefrontal and medial-temporal regions. Thus, these regions were targeted in the present research.

Moreover, all age-comparative training research using brain imaging has investigated training-related changes in encoding mode, which refer to changes in activity differences between an attempt to remember information (independent from success) and a no-memory explicit baseline (i.e., a task similar in visual complexity to the memory task that only requires simple perceptual decisions; Jones et al., 2006; Kirchhoff et al., 2012; Nyberg et al., 2003). In contrast, encoding success refers to the contrast between remembered and not-remembered information (i.e., omission errors) and thus helps to identify regions that are relevant to successful memory formation. In functional magnetic resonance imaging (fMRI), subsequent-memory (SM) effects refer to regional activation differences during encoding of trials that are later retrieved successfully and encoding of trials that are not retrieved. The present study investigates whether SM effects are altered by instruction and training in a mnemonic skill, and whether such alterations, if present, differ among children, younger adults, and older adults.

We used a cued-recall paradigm to investigate EM performance before and after mnemonic strategy instruction and training. Individuals were scanned during encoding of word pairs. We expected trainingrelated performance changes for all three age groups in comparison to age-matched controls. As SM effects focus on the relation between remembered and not-remembered information and not on the level of 
activation per se, it is conceivable that differences between remembered and not-remembered items are reduced after strategy training. This is so because activations for remembered and not-remembered information may become more similar after training, reflecting that the strategy is applied more consistently during encoding. The chief objective of the current research was to explore this hypothesis with special focus on potential differences in training-related activation patterns across the lifespan.

\section{Methods}

\section{Participants}

This study is part of a large-scale lifespan study with an initial sample of 95 children, 49 younger adults, and 165 older adults. Thirty-four persons from each age group were eligible to participate in an fMRI study after passing several screening criteria: (a) a minimum raw score of 24 on the digit symbol test (Wechsler, 1981); (b) at least 28 points on the Mini-Mental State Exam (Folstein et al., 1975); (c) a score of 30 or higher on the CES-D scale on depression (a lower score indicates risk for depression; Radloff, 1977); (d) at least 3 word pairs correctly recalled from a study list of 10 pairs. The last screening criterion was decided upon extensive piloting and was included to increase the likelihood that participants would produce enough remembered word pairs in the in-scanner memory task for the fMRI analyses.

For this study, 21 individuals had to be excluded from analysis: 16 individuals ( 2 children, 2 younger adults, and 12 older adults) due to not producing enough remembered or not-remembered items, either before or after training (i.e. less than 6 responses in a given category per run; Murphy and Garavan, 2005), 3 children due to excessive motion artifacts (i.e., more than $3 \mathrm{~mm}$ in any direction), 1 younger adult due to technical error in the scanning procedure, and 1 child based on extreme values in brain activation (i.e., above three standard deviations of the group mean). Hence, the effective sample consisted of 28 children (aged 10.30-11.93 years, $M=11.09$ ), 31 younger adults (aged 21.3925.77 years, $M=24.05$ ), and 22 older adults (aged 63.03-70.64 years, $M=66.73$ ). Assessments of crystallized (vocabulary) and fluid (perceptual speed) intelligence before training indicated that the age groups were representative of their respective cohorts showing a steep increment from middle childhood to young adulthood followed by stability from early to late adulthood in crystallized intelligence, and a steep increment during middle childhood with a peak in young adulthood and decline in old age for fluid intelligence (Table 1). All participants were right-handed, native German speakers, and did not report having neurological or psychiatric disorders. None of the participants was familiar with memory strategies or had any experience with strategy training. All were distributed randomly into either training or control groups. The training groups consisted of 16 children, 16 younger adults, and 11 older adults. The control groups comprised 12 children, 15 younger adults, and 11 older adults. The training and control groups did not differ in age, education, or general cognitive functioning (Table 1).

\section{Materials}

Lists of unrelated word pairs were used to assess EM performance. The words were concrete, highly imaginable German nouns (Brehmer et al., 2004; Hasselhorn et al., 1990; Scheithe and Bäuml, 1995). The word pairs were assembled randomly; however, we ensured that the words in the pairs were not semantically or associatively related. In addition, words forming a pair never started with the same letter or rhymed. Words used before and after training (assessment sessions) were unique and not used before, whereas words used during instruction and practice sessions were partly re-used and mixed across word pairs and lists.

In the assessment sessions, length of study lists was adjusted for each age group to attain an average task difficulty across groups at the level of 25-45\% accuracy before training to provide room for traininginduced performance gains. In addition, a sufficient number of trials for the specific fMRI analysis were needed (i.e., at least 6 responses in a given category per run). Thus, extensive piloting resulted in the following age-specific set up: Children received two lists of 72 word pairs each, younger adults received two lists of 96 word pairs each, and older adults four lists of 28 word pairs each. Four word pairs were added to each study list as filler pairs (the first two and the last two pairs) to avoid primacy and recency effects. These word pairs were not used in the fMRI analyses.

The training groups were exposed to a total of 90 word pairs during instruction and 192 word pairs during practice of the memory strategy. Although all age groups received six lists of 15 word pairs each during instruction, age-specific arrangements of list length were applied during the two practice sessions: during each session, children received three times 32 word pairs, younger adults two times 48 word pairs, and older adults six times 16 word pairs. Thus, the total number of trials was identical for all age groups, but the length of the practice lists was between $40 \%$ and $60 \%$ of the list lengths used during the assessment sessions to provide an equally challenging context across age groups.

\section{Experimental procedure}

\section{Encoding phase}

The design of the in-scanner procedure is presented in Fig. 1. Word pairs were presented visually on a computer screen for 6 seconds (one at the top, one at the bottom) followed by a question prompting for judgment of learning for 2 seconds ("how sure are you that you will later remember the word pair?"). This question phase served to ensure that participants remained attentive to the task. Participants entered their responses by pressing "very sure," "sure", or "unsure." An explicit baseline condition was included to better separate activations for memory versus non-memory processes, avoiding the problem of an ambiguous rest baseline (Stark and Squire, 2001). In the explicit baseline condition, participants saw pairs of letter strings ( $x x x x x-k k k k k$ ). Six trials were presented sequentially forming one block, each trial lasting for 1 second. In $1 / 3$ of these control blocks, there were trials where the pairs of letter strings consisted of the same letters. To ensure that participants were engaged in the low-level task, they were asked to monitor the occurrence of these trials and to press a button. In $2 / 3$ of the blocks, no button presses were required and these blocks were the explicit baseline condition for the memory task. Scanning time for the baseline task was roughly $1 / 3$ of the scanning time for the actual memory task. Memory trials and baseline blocks were presented in randomized order, each separated by a jittered fixation period, ranging from 500 to $1500 \mathrm{~ms}$ (in $500 \mathrm{~ms}$ steps).

\section{Recall phase}

After each encoding list, participants were tested using a cued-recall procedure. Participants saw one word (the one presented on top of the screen during encoding) at the top of the screen and were asked to recall the corresponding word from the pair. They indicated whether they could remember the word by pressing either "yes" or "no." If "yes" was pressed, participants reported the corresponding word followed by a confidence rating. When "no" was pressed, the next trial appeared.

During the assessment sessions, the procedure differed slightly for children compared to younger and older adults. For younger and older adults, retrieval was done inside the scanner without scanning. Based on piloting, lying in the scanner at both encoding and retrieval was too strenuous for children, potentially lowering the quality of subsequently acquired MRI data due to movement artifacts. Therefore, children's retrieval was done outside the scanner on a computer in a quiet room. Children entered the scanner again after completing retrieval of the first list. During assessment sessions, participants reported the answer verbally (in the case of younger and older adults, through the 
Table 1

Sample characteristics.

\begin{tabular}{|c|c|c|c|c|c|c|}
\hline \multirow[b]{2}{*}{ Measures } & \multicolumn{3}{|l|}{ Training } & \multicolumn{3}{|l|}{ Control } \\
\hline & $\begin{array}{l}\mathrm{CH} \\
(\mathrm{n}=16)\end{array}$ & $\begin{array}{l}\mathrm{YA} \\
(\mathrm{n}=16)\end{array}$ & $\begin{array}{l}\mathrm{OA} \\
(\mathrm{n}=11)\end{array}$ & $\begin{array}{l}\mathrm{CH} \\
(\mathrm{n}=12)\end{array}$ & $\begin{array}{l}\text { YA } \\
(n=15)\end{array}$ & $\begin{array}{l}\mathrm{OA} \\
(\mathrm{n}=11)\end{array}$ \\
\hline Age & $11.02(.37)$ & $24.21(1.32)$ & $67.13(1.99)$ & $11.18(.45)$ & $23.88(1.19)$ & $66.33(2.42)$ \\
\hline$\%$ females & $37.5 \%$ & $50 \%$ & $36.36 \%$ & $33.33 \%$ & $46.67 \%$ & $45.45 \%$ \\
\hline Years of education & $5.25(.68)$ & $21.07(1.98)$ & $21.5(4.70)$ & $5.33(.49)$ & $21.67(3.91)$ & $23.36(4.57)$ \\
\hline Digit symbol & $37.53(7.36)$ & $72.19(10.09)$ & $48.45(7.45)$ & $43.67(7.83)$ & $68.33(11.24)$ & $49.73(8.70)$ \\
\hline Vocabulary & $10.56(4.02)$ & $23.05(2.96)$ & $28.81(2.98)$ & $9.25(3.92)$ & $21.75(4.30)$ & $28.98(2.15)$ \\
\hline $\mathrm{Nr}$. of strategies at pretest & $1.50(.73)$ & $1.44(.63)$ & $1.64(.81)$ & $1.25(.45)$ & $1.53(.64)$ & $1.18(1.63)$ \\
\hline
\end{tabular}

Note. Mean (SD). $\mathrm{CH}=$ children, $\mathrm{YA}=$ younger adults, $\mathrm{OA}=$ older adults. Training and control groups did not differ in any of the above variables.

scanner speaker). In the instruction and practice sessions, participants entered their answers using the keyboard.

\section{Experimental sessions}

Table 2 summarizes the various sessions of the study. All participants took part in a first behavioral session that consisted of assessment of demographic information and a psychometric test battery. During the second session, participants were trained on getting comfortable with the MRI environment inside a scanner simulator (NordicNeuroLab). They also practiced on the task to get used to encoding word pairs while lying in the simulator. Motion was detected using a camera and feedback was given directly when head movement was detected. In the third session (pre-training assessment) participants were scanned during wordpair encoding. They were instructed to encode the words as pairs for an upcoming cued-recall test. Presentation order of word pairs within lists was randomized during encoding and retrieval to minimize the use of semantic strategies (e.g., sentence/story generation across word pairs) and to maximize the relative contribution of associative processing for each word pair. After completing the memory task, participants were asked to describe all strategies they had used for encoding the word pairs in an open format. These strategies were later on coded into three broad categories based on Dunlosky and Hertzog (1998): visual, semantic, and shallow (e.g., rote repetition) strategies.

The training groups continued with four strategy instruction and practice sessions, whereas the control groups had a break for the same amount of time. The essence of the strategy was to elaborate on the word pairs using visual imagery to integrate the two words (Paivio, 1969). The first instruction session took place in age-homogenous groups of 3-4 individuals. Participants were introduced to the main principles of the strategy using concrete examples. Several short lists word pairs were used to practice the generation of interactive and dynamic images integrating the two un-related words into one coherent memory representation. Afterwards, participants practiced the technique with two word lists on a computer individually. In the following single-instruction session, instruction and supervised training were continued individually to improve the quality of the visual images generated by the participants. This was done by (a) using prompts to verbalize and discuss all aspects of image formation and image retrieval, (b) assistance during recall, (c) repetition and elaboration of instructions, as well as various other forms of encouragement (see Brehmer et al., 2007 for a similar instruction approach). The instruction sessions lasted about 1 hour each.

Participants practiced the imagery strategy in two consecutive sessions with several lists of age-group-specific lengths (see description above). Practice sessions took place in groups of 2-5 individuals. Individuals in these groups practiced the task on separate computers at an individualized pace and without any further strategy-relevant assistance from the experimenter. The practice sessions lasted between 40 and 60 minutes each.

The final session (post-training assessment) was identical to the pretraining assessment. Trained participants were again scanned during word-pair encoding. Participants from the control groups were given the same instruction as during pre-training assessment, namely, to encode the word pairs as pairs for an upcoming cued-recall test. Participants from the training groups were instructed to use the newly acquired memory strategy to encode and later recall the word pairs. As during pre-training assessment, word pairs were presented visually on a computer screen for 6 seconds (one at the top, one at the bottom) followed by a question phase for 2 seconds. Although the control groups received the same question as during pre-training assessment, namely, prompting for judgment of learning ("How sure are you that you will remember the word pair?"), the trained groups were asked to judge the quality of the generated image for each word pair ("Were you able to generate an interactive image for this word pair?"). Participants entered their responses by pressing "no image," "a kind of image," or "interactive image."

This was done to emphasize the consistent use of interactive imagery during encoding for the trained individuals.

After completing the memory task, participants were again asked to describe all strategies they had used for encoding the word pairs in an open format.

\section{MRI protocol}

Whole-brain MRI data were collected with a Siemens $3 \mathrm{~T}$ Trio Magnetom.

\section{Functional scans}

Blood-oxygen-level-dependent (BOLD) fMRI images were generated using an echo-planar-imaging (EPI) pulse sequence $(\mathrm{TR} / \mathrm{TE}=$ $2000 / 30 \mathrm{~ms}$, flip angle $=90^{\circ}$, matrix $=72 \times 72, \mathrm{FOV}=216 \mathrm{~mm}$,

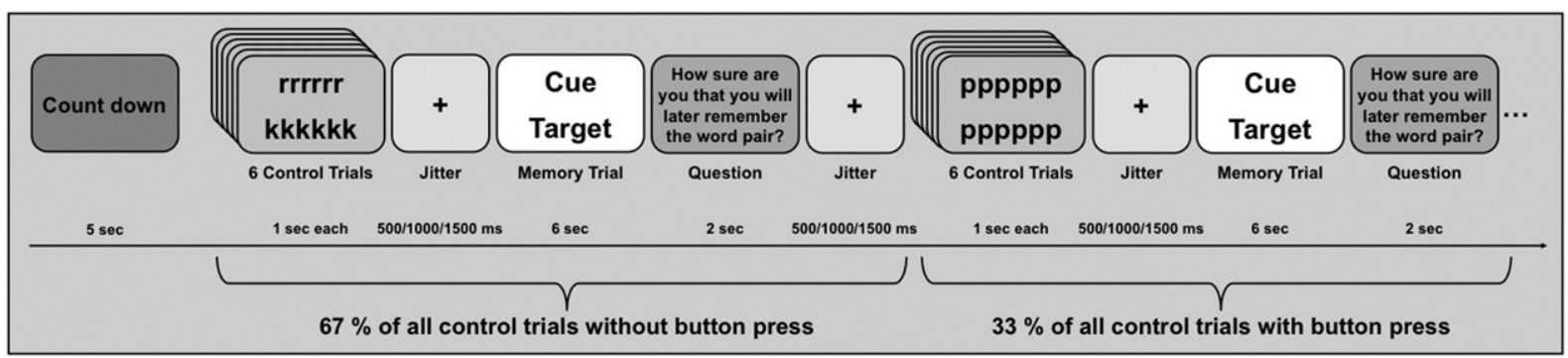

Fig. 1. Design of the associative memory task used in scanner. 
Table 2

Overview of the study design.

\begin{tabular}{lll}
\hline Study phase & Content & \multicolumn{1}{c}{$\begin{array}{l}\text { Session } \\
\text { (no.) }\end{array}$} \\
\hline Covariate session I & $\begin{array}{l}\text { Assessment of general demographic } \\
\text { information and psychometric intelligence }\end{array}$ & 1 \\
Covariate session II & $\begin{array}{l}\text { MRI simulator } \\
\text { Memory questionnaires } \\
\text { Visual and auditory acuity } \\
\text { PMRI assessment during memory encoding }\end{array}$ & 3 \\
Imagery strategy instruction & $\begin{array}{l}\text { Strategy use questionnaire } \\
\text { Group and single instruction sessions with } \\
\text { practice of word lists }\end{array}$ & $4+5$ \\
Imagery strategy practice & $\begin{array}{l}\text { Several practice lists } \\
\text { fMRI assessment during memory encoding }\end{array}$ & 8 \\
\hline Post-training assessment & Strategy use questionnaire & \\
\hline
\end{tabular}

Note. Training groups participated in all sessions, control groups performed sessions 1-3 and 8 only.

voxel size $=3 \times 3 \times 3 \mathrm{~mm}^{3} ; 36$ slices). Slices were acquired interleaved in axial orientation. Three dummy scans were performed prior to image acquisition to eliminate signals arising from progressive saturation. Before acquisition of each functional sequence, a new localizer was acquired to adjust the scanning window (alignment based on genusplenium of the corpus callosum).

\section{Structural scans}

For registration of functional images, 2 structural sequences were collected, one T2-weighted turbo-spin echo sequence (TR/TE $=8170$ / $93 \mathrm{~ms}$; matrix $=256 \times 256$; in-plane resolution $=1 \times 1 \mathrm{~mm}$, slice thickness $=3 \mathrm{~mm}$ ) in the same orientation as the functional sequences; and one high-resolution T1-weighted MPRAGE sequence (TR/TE = $1550 / 2.34 \mathrm{~ms}$; matrix $=256 \times 256$, in-plane resolution $=1 \mathrm{~mm}$, slice thickness $=1 \mathrm{~mm}$ ). Gradient echo images were measured for correction of magnetic field inhomogeneities.

\section{Data analyses}

\section{Functional brain activity}

Preprocessing. A quality check of the functional data was conducted using dataQuality, a Matlab-based tool (http://cbi.nyu.edu/software/ dataQuality.php), which identifies spikes (signals greater than 10 standard deviations above the mean (across time) and corrects these by substituting the value with an average of the intensity of the two time points before and after the problematic time point.

Data for each run from each participant were analyzed using FEAT in FSL (Version 6.00, FMRIB's Software Library, http://www.fmrib.ox.ac. uk/fsl, Smith et al., 2004). Before images were subjected to preprocessing, BET (Brain Extraction Tool; Smith, 2002) was used to strip away the skull and other non-brain parts of the images. Images were motion corrected using rigid body transformation as implemented in MCFLIRT (Motion Correction using FMRIBs Linear Image Registration Tool; Jenkinson, Bannister, Brady, \& Smith, 2002), and smoothed using an isotrophic $8.0 \mathrm{~mm}$ full-width half-maximum Gaussian filter to remove low-frequency noise. Data with motion more than $3 \mathrm{~mm}$ in any direction were handled by either removing that particular run entirely or leaving out the volumes (and subsequent volumes) affected by motion if it occurred toward the end of the measurement. During pretraining assessment, this affected 2 children ( 1 had one run removed, 1 had specific volumes removed), whereas none was affected during post-training assessment. A pre-whitening technique was used to account for the intrinsic temporal autocorrelation of BOLD imaging. Low-frequency artifacts were removed by applying a high-pass temporal filter (Gaussian-weighted straight-line fitting, sigma $=50$ seconds). Age-group-specific brain templates were created from participants' high-resolution images using the non-linear registration ANTS program
(Avants et al., 2008, 2011), following the iterative procedures of Sanchez et al. (2012). Functional scans were registered to their own high-resolution scans, then to the age-specific brain templates, and finally to standard space (MNI) using FLIRT (Jenkinson et al., 2002).

fMRI data analysis. Analysis of the imaging data was performed according to the general linear model. First-level individual analyses modeled the time series with separate regressors for subsequently remembered, not remembered, and incorrectly remembered trials for each run and MRI session (pre-training and post-training). Additional regressors included the question phase, the explicit baseline phase (with and without button presses, separately), and filler pairs at the beginning and end of the run. The regressors were generated by convolving the impulse function related to the onsets of events of interest with a Gamma hemodynamic response function (HRF). The critical contrast for the SM effect was [remembered $>$ omissions or R $>0$ )] . Contrast images were computed for each run per subject, spatially normalized, transformed into MNI standard space, and submitted to a withinsubject fixed-effects analysis across runs for pre- and post-training, separately. To estimate activation changes across time, these contrasts were then compared between pre- and post-training on an individual level using fixed effects. These individual time contrasts were entered into a higher-level analysis across subjects using a mixed-effects model in FSL (FLAME, Woolrich et al., 2004). All brain coordinates are reported in MNI space.

The first analysis tested age-general training-induced changes in BOLD activity for the different experimental groups in task-relevant regions. Task-relevant regions were identified as follows: Within each age and experimental group (i.e., $\mathrm{CH}_{\text {Train }}, \mathrm{CH}_{\mathrm{Con}}, \mathrm{YA}_{\text {Train }}, \mathrm{YA}_{\text {Con }}, \mathrm{OA}_{\text {Train, }}$, $\left.\mathrm{OA}_{\text {Con }}\right)$, we computed the condition contrast $(\mathrm{R}>\mathrm{O})$ separately for pre- and post-training assessments. Based on these contrast maps of each age group, we computed a disjunction map ( $p<.01$, uncorrected). Thus, the disjunction image showed activation in regions in which any of the six groups showed SM effects either at pre- or post-training assessment. By using this disjunction image, we avoid the problem of biasing the selection of voxels with regard to the age/experimental condition manipulation of interest here (non- independence errors; Kriegeskorte et al., 2009) and maximize the probability of finding activation changes in regions relevant before and/or after training. In a GLM group analysis, the time contrast $\left([\mathrm{R}>0]_{\text {pre-training }}\right.$ versus $[\mathrm{R}>\mathrm{O}]_{\text {post-training }}$ ) of the training and control groups was compared, controlling for exact age (as an indirect control of age-related differences in list length), using the disjunction image as the only whole-brain restriction. Functional activation was thresholded at a significance level of $p<.01$ ( $Z>2.3$, uncorrected) and reported effects show a cluster volume of at least 54 contiguous voxels, as determined by a Monte Carlo simulation for minimum cluster size where the probability of type I error was $<.05$ (https://www2.bc.edu/ slotnics/scripts.htm; Slotnick, 2003).

To investigate whether training-induced changes in SM effects were comparable across age or specific to one or the other age group, we defined ROIs by placing a $3-\mathrm{mm}$ sphere around the peak activation in those brain regions showing a time $\times$ treatment interaction in the GLM group analysis (for MNI coordinates of ROIs, see Table 4). From these ROIs, mean percent signal change was extracted per subject for SM effects pre- and post-training separately for each ROI. Percent signal changes were analyzed with age $\times$ time repeated-measures ANOVAs for the trained individuals. A main effect of time without a time $\times$ age interaction would indicate similar changes for all three age groups. However, a time effect and a time $\times$ age interaction would reflect that the groups differed in degree of change. When this was the case, pair-wise comparisons were conducted to specify which group was driving the effect. Corresponding analyses were conducted for the control groups to ensure that the observed findings were unique to the trained groups and not related to repeated testing.

Third, an ROI-based approach was applied to investigate potential age-related differences in training-induced changes in SM effects in 
brain regions known to be most critical in supporting successful encoding (i.e., left BA9, BA44/45 and BA46, bilateral hippocampus (HC) and parahippocampal gyrus (PHG); see Kim, 2011; Spaniol et al., 2009). First, anatomical ROIs of these regions were delineated using the WFU PickAtlas (Maldjian et al., 2003, 2004) for the frontal regions and the Harvard-Oxford Cortical and Subcortical Structural Atlas (http://www.cma.mgh.harvard.edu/fsl_atlas.html), with voxels thresholded at a minimum $25 \%$ probability of being within a specific region for $\mathrm{HC}$ and PHG. To ensure that only task-positive regions in these ROIs were entered into the analysis, a conjunction was made between the anatomical ROIs and the map of SM effects $(R>0)$ of taskpositive brain regions in any of the six age/experimental groups at either pre- or post-training assessments. Here, functional activation was again determined by thresholding $Z$ (Gaussianized T/F) statistics image using clusters determined by $\mathrm{Z}>2.3$, uncorrected ( minimum cluster size $=54$ contiguous voxels). Percent signal changes of SM effects were extracted for each individual before and after training. These signal changes were subjected to repeated-measures ANOVAs, with age as between-subjects factor and time (pre- and post-training assessment) as within-subjects factor. To ensure that these effects were training-induced and not evoked by repeated testing, a similar analysis was again conducted for the control groups. Effects were considered significant at an alpha level of .05 .

\section{Results}

\section{Behavioral data}

\section{Cued recall of word pairs}

As noted, list length of each age group was adjusted to achieve an accuracy level between $25 \%$ and $45 \%$ before training. Nevertheless, an ANOVA with age and experimental condition as between-subjects factors before training revealed a significant effect of age $F(2,75)=6.25$, $p<.01, \eta^{2}=.14$, indicating that children $(M=.25, S E=.03)$ remembered fewer word pairs than younger adults $(M=.40, S E=.04)$, $p<.01$. However the difference between children and older adults $(M=.32, S E=.03)$ and between older and younger adults did not reach statistical significance ( $p s>.08$; see Table 3 ). Furthermore, pretraining performance of the training and control groups was indistinguishable $(F<1)$.

A repeated-measures ANOVA with age and experimental condition as between-subjects factors and time as a within-subjects factor was conducted to investigate if strategy training resulted in greater improvement than repeated testing. The main effects of experimental condition $\left(F(1,75)=11.58, p<.01, \eta^{2}=.13\right.$, age $(F(2,75)=10.20$, $\left.p<.001, \eta^{2}=.21\right)$, and time $F(1,75)=181.90, p<.001, \eta^{2}=.71$ reached significance. Further, the experimental condition $\times$ time interaction was significant, $F(1,75)=81.21, p<.001, \eta^{2}=.52$, indicating larger gains from pre- to post-training for the trained groups than the controls. However, the age groups did not differ in degree of change $(p>.11)$ and the age $\times$ experimental condition $\times$ time interaction felt far short of significance ( $p>.26$; Fig. 2$)$.

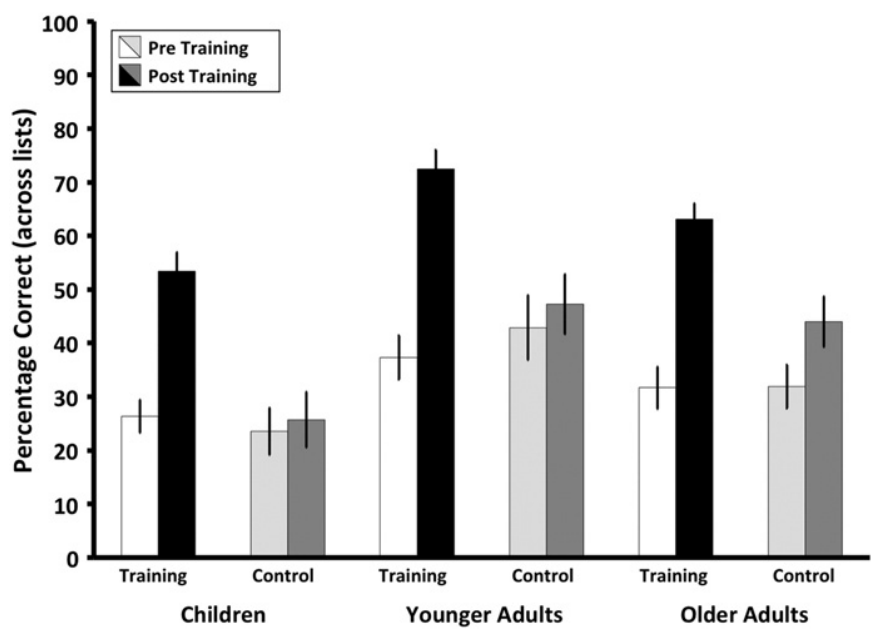

Fig. 2. Performance changes from pre- to post-training sessions. Trained individuals showed larger performance gains compared to controls, independent of age. Percentage correct (across lists) for children, younger adults, and older adults in training and control groups. Pre-training performance is given in white/light gray and post-training performance is depicted in black/dark gray. Error bars represent standard errors.

\section{Strategy use}

To investigate the effectiveness of strategy instruction and practice, we examined how the different age and intervention groups reported encoding the word pairs at pre- and post-training assessments (Fig. 3). Reported strategies were sorted into visual, semantic, or shallow. The most commonly used strategy before training was semantic $(M=.65, S E=.05)$, followed by visual $(M=.23, S E=.04)$, and shallow $(M=.12, S E=.03)$ strategies. Significant age effects were observed for visual and shallow strategies, reflecting that younger adults reported more use of visual strategies than children $(p<.05)$, whereas younger and older adults did not differ $(p>.10)$. Children reported using more shallow strategies than both adult groups ( $p s<.05$ ), which again did not differ $(p>.10)$. Importantly, training and control groups did not differ in strategy use before training $(p=.39)$. Regarding training-related strategy changes, experimental condition $\times$ time interactions were observed for all three types of strategies ( $p s<.05$ ). To trace the sources of these effects, separate analyses for the training and control groups were conducted. For the training groups, time effects were observed for semantic and visual strategies ( $p s<.001$ ), reflecting an increase for visual strategies and a decrease for semantic strategies. A trend toward a significant age $\times$ time interaction was observed for shallow strategies $(p=.054)$, suggesting that children showed more decreases in using shallow strategies than younger adults (no shallow strategies were reported by older adults before or after training). No main effect of time and no age $\times$ time interaction were observed for the control groups.

\section{fMRI data}

General training-induced changes in the subsequent-memory effect

In the first fMRI analysis, training-induced changes in SM effects were investigated in task-positive brain regions comparing trained

Table 3

Number of remembered items and omissions at pre- and post-training assessments across age and experimental groups.

\begin{tabular}{|c|c|c|c|c|c|c|}
\hline & \multicolumn{3}{|l|}{ Training } & \multicolumn{3}{|l|}{ Control } \\
\hline & $\mathrm{CH}$ & YA & $\mathrm{OA}$ & $\mathrm{CH}$ & YA & $\mathrm{OA}$ \\
\hline Total number of trials & 144 & 192 & 112 & 144 & 192 & 112 \\
\hline Remembered trials T1 & $35.81(25 \%)$ & $70.44(37 \%)$ & $35.45(32 \%)$ & $32.58(23 \%)$ & $80.73(42 \%)$ & $35.45(32 \%)$ \\
\hline Omissions T1 & $96.94(67 \%)$ & $107.25(56 \%)$ & $68.09(61 \%)$ & $94.83(66 \%)$ & $101.20(53 \%)$ & $69.09(62 \%)$ \\
\hline Remembered trials $\mathrm{T} 2$ & $72.81(51 \%)$ & $135.06(70 \%)$ & $70.27(63 \%)$ & $36.17(25 \%)$ & $90.07(47 \%)$ & $48.64(43 \%)$ \\
\hline Omissions T2 & $54.50(38 \%)$ & $42.75(22 \%)$ & $31.09(28 \%)$ & $96.75(67 \%)$ & $94.67(49 \%)$ & $56.91(51 \%)$ \\
\hline
\end{tabular}

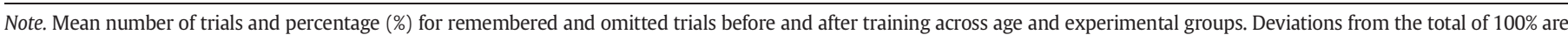
based on small numbers of incorrectly remembered trials, which were not used in the subsequent-memory analysis. $\mathrm{CH}=$ children. YA $=$ younger adults. OA $=$ older adults. 

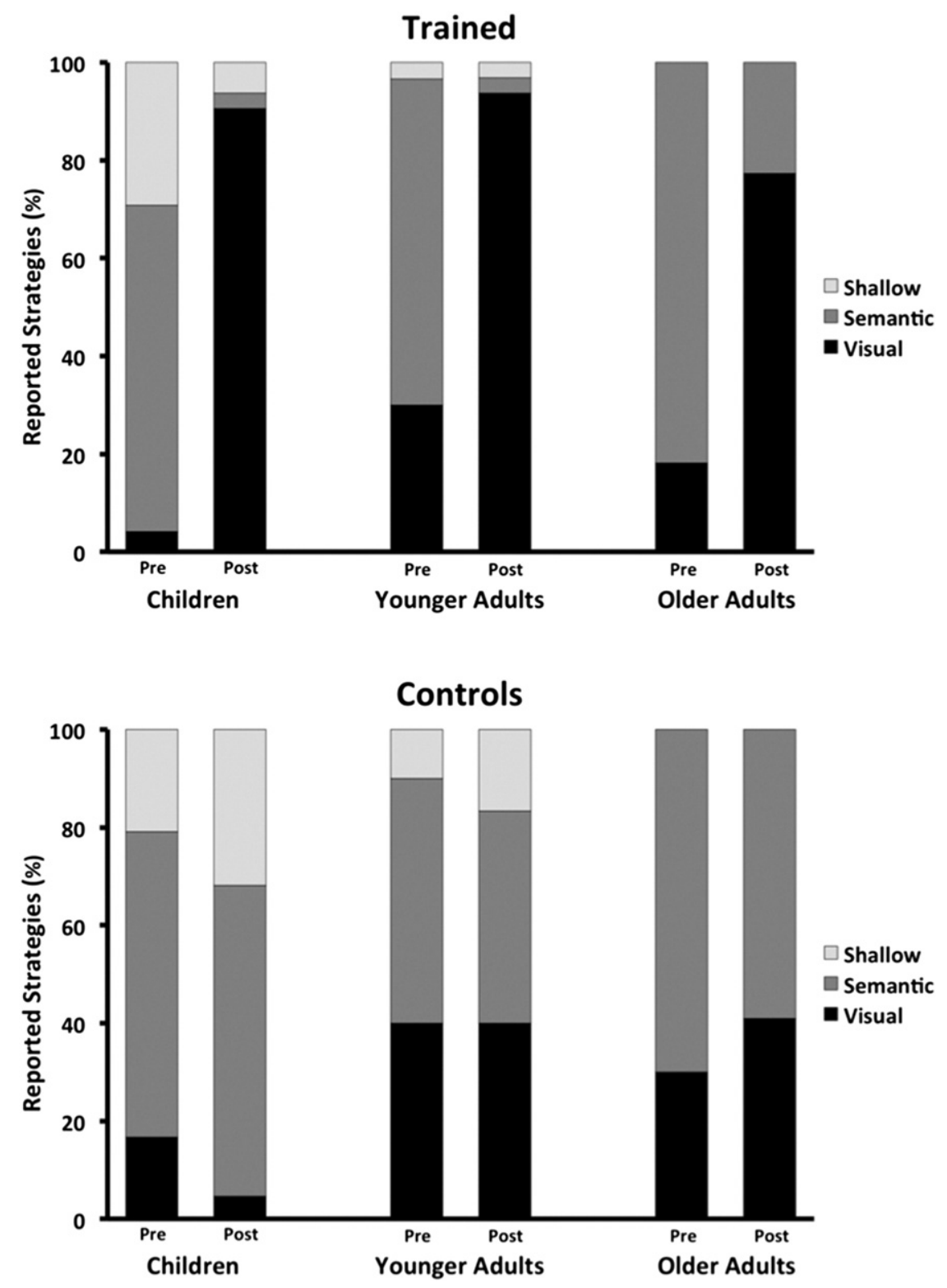

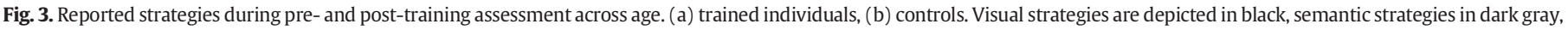
and shallow strategies in light gray. Trained individuals increased in visual strategy use across age-no changes were observed for the control groups.

individuals with controls (across all groups, controlling for exact age). Overall, the training groups showed intervention-related decreases in SM effects in several brain areas, including left frontal (inferior, middle), left temporal (inferior, middle, superior), right inferior temporal, left parietal (precuneus), bilateral occipital (cuneus), left middle occipital, and left HC and PHG regions. No training-related activity increases for SM effects were observed. A direct comparison with the control groups revealed training-specific changes in SM effects in left frontal regions (i.e., inferior (BA45), middle (BA9), and medial (BA6; see Table 4 for details)). Possible age-related differences in training-induced changes in the SM effect in these regions were investigated next.

Age differences in training-induced changes in the subsequent-memory effect

All three age groups exhibited comparable decreases in the SM effects for the left inferior frontal (BA45) and the left middle frontal (BA9) regions $(p s<.05)$. Only the left medial frontal (BA6) region showed an age $\times$ time interaction $(p<.05$; Fig. 4$)$. Follow-up analysis revealed that this effect was mainly driven by older adults' greater reduction in the SM effect compared to that of younger adults $(p<.05$; for the difference in reduction between older adults and children, $p=.063)$. In the same region, there was also a trend for agegroup differences in the SM effect at baseline $(p=.09)$, suggesting a larger SM effect in older adults before training in comparison to younger adults and children, which disappeared at post-training assessment $(p>.32)$.

Activation changes underlying the training-induced reduction in the subsequent-memory effect

To examine whether reductions in SM effects were due to activation changes for remembered items, not-remembered items, or both, percent signal change was extracted from the relevant frontal regions (using spheric ROIs with a $3 \mathrm{~mm}$ radius) for the contrasts remembered items [ $\mathrm{R}>$ explicit baseline] and omissions [ $\mathrm{O}>$ explicit baseline], separately for pre- and post-training assessments. Using repeated-measures ANOVAs with age as between-subjects factor and time as a withinsubjects factor for remembered items and omissions, we investigated 
Table 4

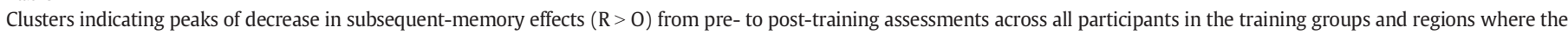

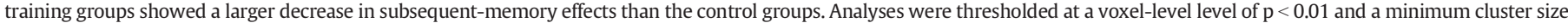
of 54 contiguous voxels.

\begin{tabular}{|c|c|c|c|c|c|c|c|}
\hline \multirow[b]{2}{*}{ Brain area } & & \multicolumn{6}{|l|}{ Training group } \\
\hline & & $\mathrm{BA}$ & $\mathrm{Z} \max$ & $\mathrm{X}$ & $\mathrm{Y}$ & Z & No. of voxels \\
\hline Inferior, middle frontal & Left & $10,13,46$ & 4.6 & -44 & 44 & -6 & 7408 \\
\hline Inferior, middle, superior temporal & Left & $20,21,37,38$ & 4.75 & -60 & -42 & -6 & 1281 \\
\hline Inferior temporal & Right & 20 & 3.71 & 64 & -36 & -20 & 83 \\
\hline Precuneus, parietal & Left & 7 & 3.41 & -18 & -80 & 42 & 128 \\
\hline Cuneus, occipital & $\begin{array}{l}\text { Left/ } \\
\text { Right }\end{array}$ & $17 / 18$ & 3.23 & -6 & -88 & 18 & 137 \\
\hline Middle occipital & Left & 18 & 2.94 & -18 & -86 & 18 & 68 \\
\hline \multirow[t]{2}{*}{ Cerebellum, HC, PHG } & Left & n.a. & 3.25 & -26 & -34 & -8 & 362 \\
\hline & & \multicolumn{6}{|c|}{ Training $>$ Control } \\
\hline Brain area & & $\mathrm{BA}$ & $\mathrm{Z} \max$ & $\mathrm{X}$ & $\mathrm{Y}$ & Z & No. of voxels \\
\hline Inferior frontal & Left & 45 & 3.21 & -44 & 16 & 8 & 303 \\
\hline Middle frontal & Left & 9 & 2.76 & -44 & 16 & 26 & 87 \\
\hline Medial frontal & Left & 6 & 2.67 & -2 & 18 & 44 & 106 \\
\hline
\end{tabular}

Note. Coordinates $\mathrm{X}, \mathrm{Y}, \mathrm{Z}$ are reported in MNI space.

which activation changes were driving the training-related alterations in SM effects (Fig. 5).

The reduction of the SM effect in the left inferior frontal region was driven by greater activation increases for omissions than for remembered items after training $F(1,40)=6.97, p=.012, \eta^{2}=.15$. A trend in the same direction was observed in middle frontal gyrus, $F(1$, $40)=3.55, p=.067, \eta^{2}=.08$. This indicates that the activations for omissions were more similar to those for remembered items after instruction and practice in the mnemonic strategy. In contrast, the reduction of the SM effect in left medial frontal regions was primarily driven by activation decreases for remembered items compared to omissions, $F(1,40)=10.22, p=.003, \eta^{2}=.20$. No three-way interactions involving changes for remembered items, changes for omissions, and age were observed, indicating that such changes, when observed, did not differ reliably among children, younger adults, and older adults.

Age-related differences in training-induced changes in subsequent memory in PFC and MTL: ROI analyses

Next, focusing the analysis on regions known to be critical to EM functioning, we investigated potential age-related differences in training-induced changes in SM effects targeting left PFC and bilateral MTL, using a conjunction of anatomical and functional masks. In accordance with the findings reported above, only training-related reductions in SM effects were observed in this analysis (see Fig. 6). Children, younger adults, and older adults showed similar decreases in SM effects for left BA44/45 and left BA46 as well as for left HC and PHG (main effects of time, $p s<.05$ ). However, in addition to overall time effects, an age $\times$ time interaction was observed for left BA 9 , reflecting that older adults showed greater reductions in the SM effect than children and younger adults $(p s<.04)$. At post-training assessment, older adults showed a significantly smaller SM effect in comparison to children and younger adults $(p s<.04)$. No significant time effects were observed for right $\mathrm{HC}$ and right PHG. No main effect of time and no age $\times$ time interaction were observed in the control groups. To investigate whether activation changes for remembered items, omissions, or both, accounted for the reductions in SM effects, repeated-measures ANOVAs with age as between-subjects factor and time as a withinsubjects factor for remembered items and omissions were conducted for those pre-defined EM ROIs, which showed a significant decrease in the SM effect from pre- to post-training. Percent signal change was extracted for the contrasts remembered items [ $\mathrm{R}>$ explicit baseline] and omissions [ $\mathrm{O}>$ explicit baseline] separately for pre- and post-training assessments. Overall, activation for remembered items and omissions increased from pre- to post-training assessments (see Fig. 7). However, even though the interaction between remembered items and omissions did not reach statistical significance $(p>.05)$, the patterns are in line with the previous analysis, indicating that activation increases were larger for omissions than remembered items. The larger decrease of the SM effect for older adults compared to children and younger adults seems to reflect that older adults' increases for omissions were larger than those for remembered items (see Fig. 8). However, this effect did not reach conventional significance $(p>.05)$.

\section{Discussion}

We examined training-induced changes in SM effects in children, younger adults, and older adults. Individuals were scanned while encoding lists of word pairs and instructed to remember the items as pairs (before training) or to apply an associative memory strategy following instructions and practice (after training). Across age, trained participants showed greater performance gains from pre- to posttraining than controls. Further, trained individuals reported using more visual associative strategies after training.

There were training-induced reductions in the SM effect in left frontal regions (inferior (BA45), middle (BA9) and medial (BA6)). These regions have been linked to generation/selection of new representations (inferior PFC) and to the ability to organize multiple pieces of information, enhancing memory for item associations (middle PFC; Blumenfeld and Ranganath, 2007; Park et al., 2013). Reductions in the SM effect in inferior frontal and middle frontal regions were comparable across age groups, although older adults showed greater reductions in the SM effect in left medial frontal cortex (BA6) than the other age groups.

These training-related changes in SM effects were primarily due to greater activation increases for omissions than for remembered items across all age groups. ROI-based analyses on brain regions known to be critical to EM functioning (i.e., prefrontal and medial-temporal) extended these findings demonstrating that children, younger adults, and older adults showed similar reductions in the SM effect for left BA44/45 and BA46 as well as for left HC and PHG. However, older adults showed greater training-related reductions in the SM effect for left BA9 than younger adults and children. In general, these regions have been found to be relevant in previous work on the influence of strategy training on neural activity (Jones et al., 2006; Nyberg et al., 2003).

\section{Similar performance gains across age groups}

In agreement with some age-comparative research indicating comparable training gains across adulthood (Bherer et al., 2006; Derwinger et al., 2003; Nyberg et al., 2003), we observed similar 


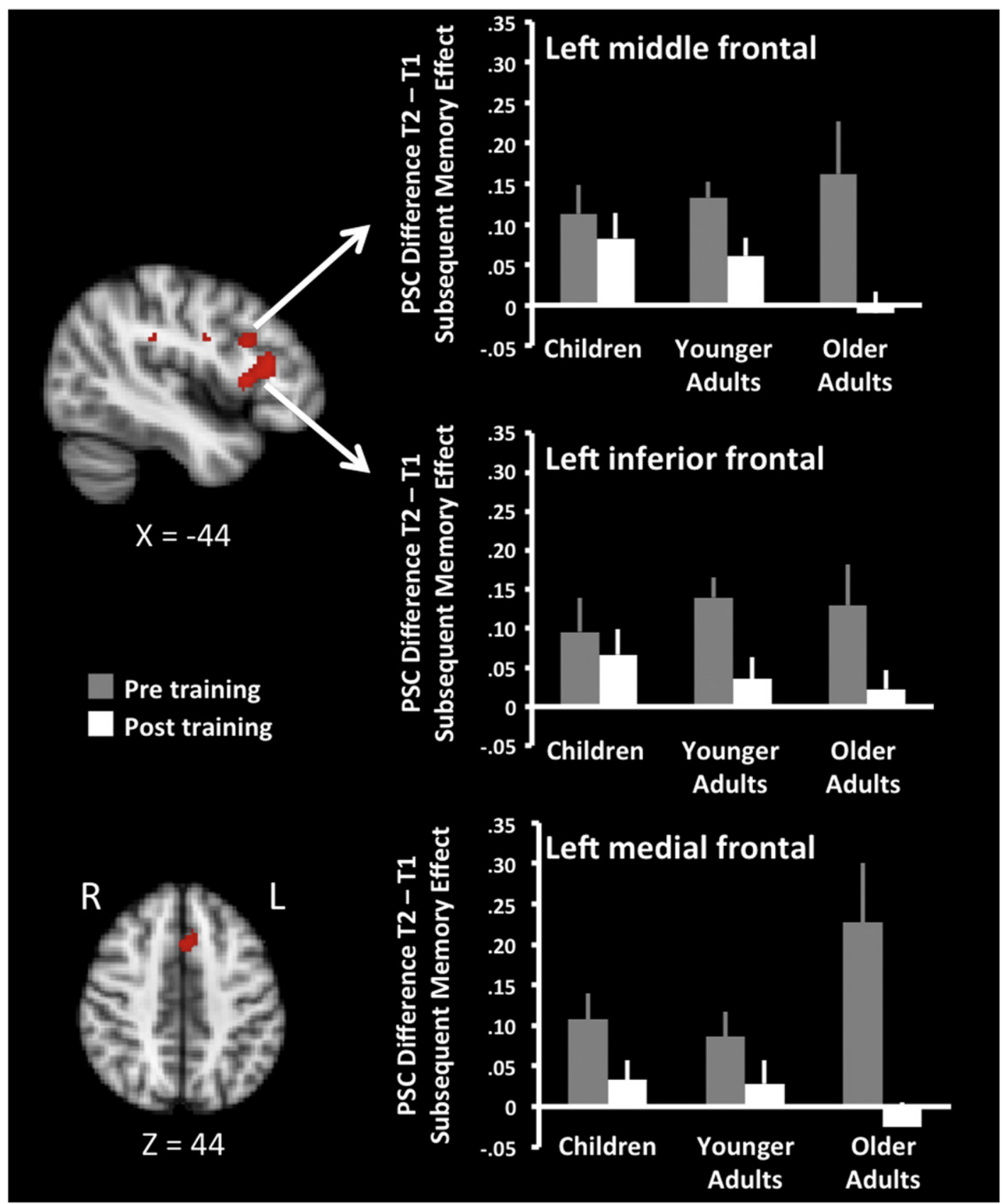

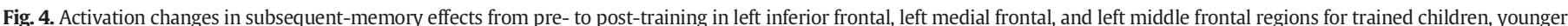

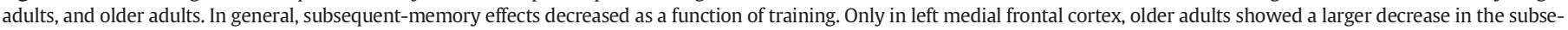

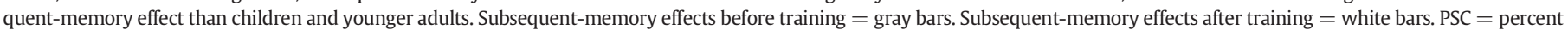
signal change. Error bars represent standard errors.

performance increments following training for all age groups. At first glance, these findings seem to be at odds with results from our previous research (Brehmer et al., 2007). In that study, children, younger adults, and older adults were instructed and trained in a variant of the method of loci. Instruction-related gains were comparable for children and older adults, although larger gains were observed for children compared to older adults after up to six sessions of adaptive training. In Brehmer et al. (2007), the adaptive training targeted the associative binding process by reducing the encoding time available to form an interactive image between to-be-remembered words and location cues during up to six sessions of practice. By contrast, the current study included only two practice sessions with a constant encoding time of 6 seconds and is, therefore, more comparable to the strategy instruction phase than the adaptive training phase used in previous research. This lack of adaptive practice might have strengthened the relative importance of strategy use and lowered the relative importance of pure associative binding processes in remembering the word pairs. In addition, note that the samples in this study were selected on the basis of associative memory performance prior to training, as one of the inclusion criteria was the ability to remember at least three out of 10 word pairs, to allow for sensitive analyses of the fMRI data. This positive selection, especially for older adults, might contribute to their high level of visualassociative strategy use at pre-training assessment, as well as their marked training-related performance gains. Before training, children and older adults as well as younger and older adults did not differ reliably from each other in task performance, but children remembered fewer word pairs than younger adults. It is worth noting that children, in contrast to younger and older adults, retrieved the to-be-learned 


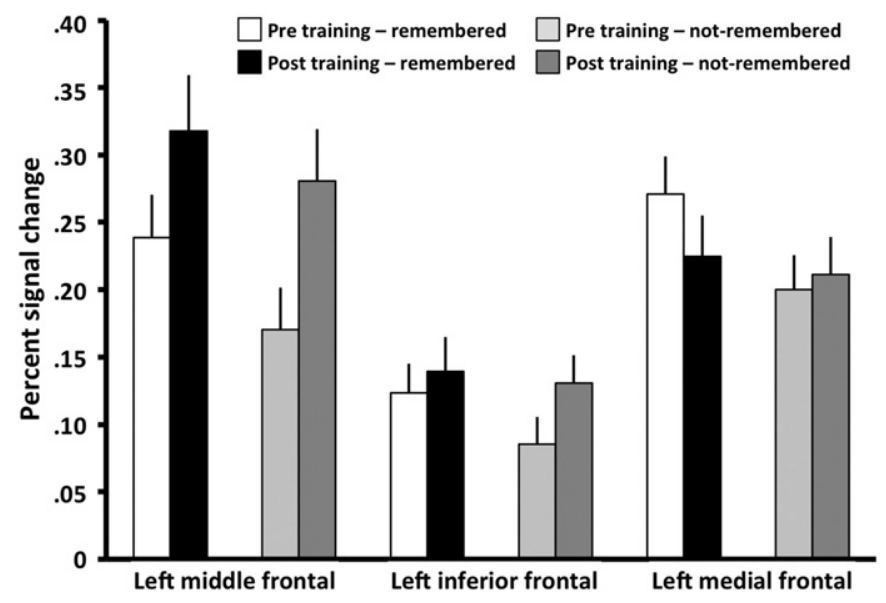

Fig. 5. Activation for remembered items [ $\mathrm{R}>$ explicit baseline] and omissions [ $\mathrm{O}>$ explicit baseline] pre- and post-training for the trained groups across age. Whereas reductions of SM effects in inferior and middle frontal regions reflected a larger activity increase for not remembered compared to remembered word pairs after training, the reduction of the SM effect in medial frontal regions reflected a decrease in activity for remembered word pairs. Error bars represent standard errors.

words outside the scanner. This was done to reduce the time of lying still, and hence movement artifacts, in the group of children. Thus, we cannot exclude that some portion of the performance difference between children and younger adults reflects differences in retrieval context.

Comparable training-induced reductions in SM effects across the lifespan

The main finding of this study is that instruction and practice in an associative memory strategy lead to reductions in SM effects, primarily in left prefrontal regions, that are independent of age. Hence, training an associative memory strategy may involve similar brain mechanisms in children, younger adults, and older adults when investigating a finegrained contrast, namely, encoding success (see Mark and Rugg, 1998; Li et al., 2004; Wang et al., 2015 for similar findings regarding retrieval success). The reductions in SM effects indicate that activations for later on remembered and not-remembered items during encoding became more similar as a function of training. This pattern primarily reflected greater activation increases for omissions compared to remembered items. Conceivably, this indicates a more consistent application of the trained strategy at post-training assessment, irrespective of recall success. This interpretation is in line with the training-related increase in the reported use of visual-associative strategies in all age groups. Note that in this age-comparative study, children, younger adults, and older adults had different numbers of word lists with different lengths (children $=2 \times 72$ word pairs, younger adults $=2 \times 96$ word pairs, and older adults $=4 \times 28$ word pairs). This procedure was decided after extensive piloting with the aim to avoid ceiling and floor effects at pre- and post-training assessments and to reach
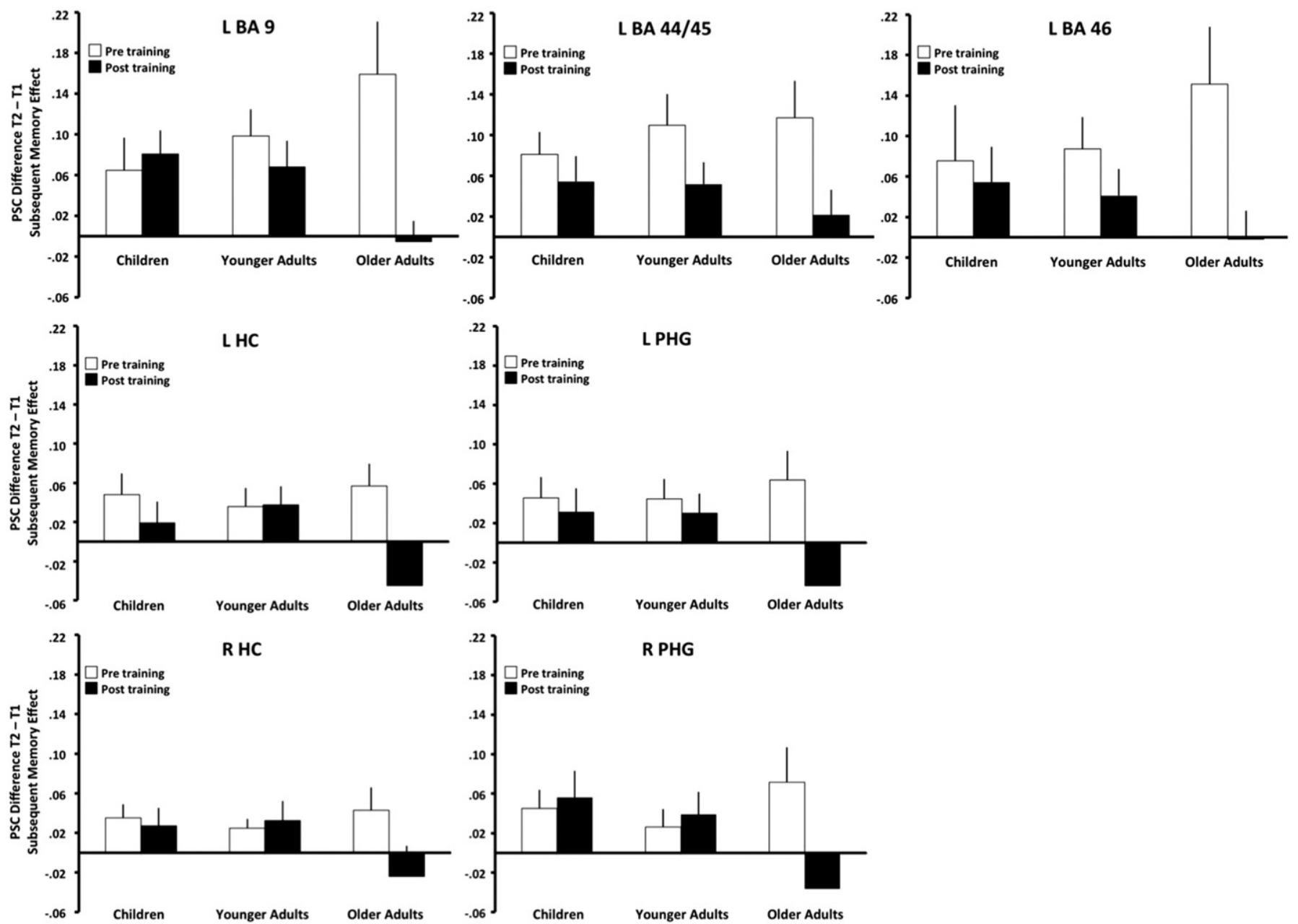

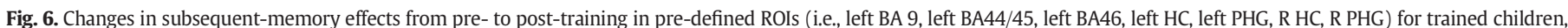

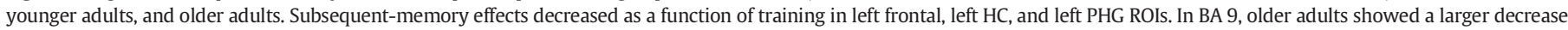

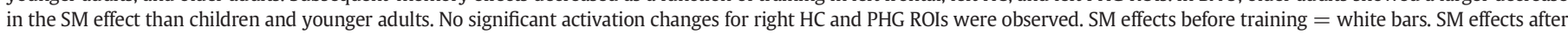
training $=$ black bars. PSC $=$ percent signal change. Error bars represent standard errors of the mean. 

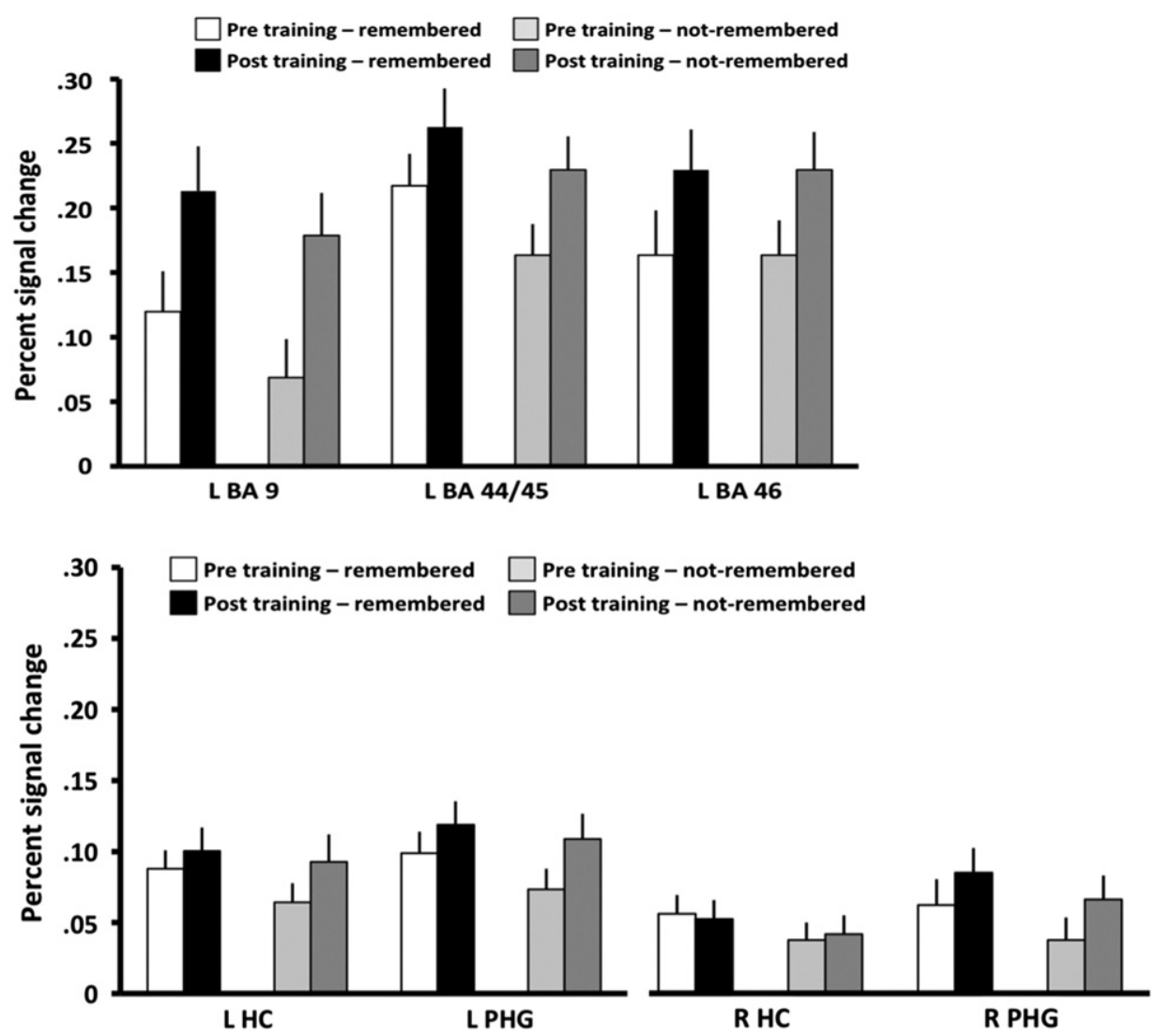

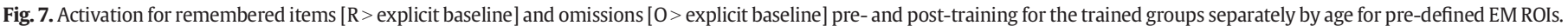
$\mathrm{L}=$ left, $\mathrm{R}=$ right, $\mathrm{BA}=$ Brodmann area, $\mathrm{HC}=$ Hippocampus, $\mathrm{PHG}=$ Parahippocampal gyrus. Error bars represent standard errors.

comparable performance levels at before training. Even though we controlled indirectly for the difference in total number of word pairs by including age as covariate into the brain analysis, future studies should try to equalize the to-be-remembered material for the different age groups even better.

The present findings shed new light on the brain maintenance hypothesis of cognitive aging (Lindenberger, 2014; Nyberg et al., 2012). Originally, this hypothesis has been formulated at the between-person level and refers to the notion that older adults with more "youth-like"

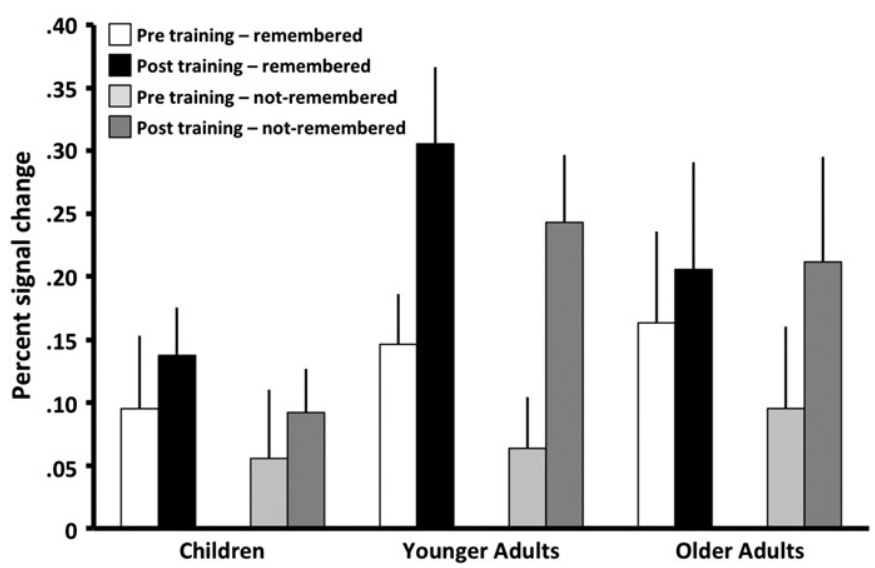

Fig. 8. Activation for remembered items [ $\mathrm{R}>$ explicit baseline] and omissions [ $\mathrm{O}>$ explicit baseline] before and after training for the trained groups separately for children, younger adults, and older adults in the pre-specified inferior frontal episodic memory ROI (BA9). A non-significant pattern suggests larger activation increases for omissions than for remembered items in older adults, while the activation increases for omitted and remembered items were more comparable in children and younger adults. Error bars represent standard errors of the mean. brains are expected to show patterns of performance and functional activation that are more similar to those of younger adults than agematched peers whose brains show greater signs of aging-related deterioration (Fandakova et al., 2015; Pudas et al., 2013). The current findings suggest that a generalization of this hypothesis also serves to explain variations within subjects. According to the generalized version, normal aging reduces the likelihood that the brain's activity is configured in ways that support successful task performance, such as successful associative encoding, without modifying the target activation pattern as such. According to this view, brains that have undergone greater decline in task-relevant regions are less likely to attain a state that is germane to successful encoding, and brains that have undergone smaller decline are more likely to attain that state, with the target state itself being largely comparable across age.

Exploring age differences in accessibility versus availability

After instruction and practice in an associative memory strategy, memory failures are less likely to reflect inadequate encoding and more likely to reflect poor consolidation, retrieval failure, or both (Cohn et al., 2008; Hertzog et al., 2013). The cued-recall paradigm used in this study is highly demanding regarding accessibility of information, but less informative when it comes to availability of information (Habib et al., 2007). Thus, future studies should combine cuedrecall and recognition paradigms to distinguish between remembered (recalled and recognized), inaccessible (not recalled but recognized), and forgotten (not recalled and not recognized) information in lifespan samples.

Select and small age group differences of training-induced reductions in SM effects

In general, the results of this study suggest that the brain areas supporting successful memory encoding following strategy instruction 
and practice remain rather stable across large portions of the lifespan. The only age-related difference observed in this study was that older adults showed greater decrease in the SM effect in left medial frontal (analysis of task-relevant areas) and left inferior frontal (analysis on encodingsuccess-specific ROIs) regions than the other age groups. Apparently, these regions were less relevant in distinguishing between remembered items and omissions after instruction and practice in an associativememory strategy for older adults. This statement applies when comparing the older adults after training to their pre-training activity (left medial frontal) and in comparing the older adults to children and younger adults after training (left inferior frontal). In the case of medial frontal regions, the greater reduction in the SM effect for older adults was accompanied by a trend for a larger SM effect before training than for younger adults and children (see Fig. 4). As this region has been linked to self-initiated use of semantic encoding strategies (e.g., sentence generation; Kirchhoff et al., 2012), it is conceivable that this region was particularly memoryrelevant for older persons before training. This interpretation is supported by the fact that older adults mostly reported semantic strategy use before training (Fig. 3). However, this effect was diminished after training. In fact, as with younger adults and children, no significant SM effects were observed here for older adults after training, indicating that this region was less critical in distinguishing between remembered and not remembered information, underscoring the effectiveness of our strategy instruction and practice. This finding is in accordance with results from a recent meta-analysis (Maillet and Rajah, 2014), which reported over-recruitment in the medial frontal cortex in contrast to younger adults, for whom this region was consistently involved in unsuccessful encoding (i.e., greater activity for omissions than remembered items). Interestingly, the over-recruitment of older adults at pre-training assessment was reduced after training in this study.

In all three age groups, reductions of the SM effect in medial frontal cortex reflected reduced activity for remembered items. Again, this finding suggests that medial frontal cortex was less important for successfully remembering word pairs after instruction and training in a visual associative memory strategy. The observation that older adults showed greater reductions in SM effects than children and younger adults in left BA 9 (ROI-based analysis on pre-defined EM regions) is surprising-as this finding suggests this region to be less important for successfully remembering word pairs after instruction and training in older adults in comparison to younger adults and children (see Fig. 6). Investigating the training-induced change for remembered and omitted trials separately, the pattern (even though not reaching statistical significance) suggests that the age-related difference in reduction in SM effect is based on larger activation increases for omissions than remembered items in older adults in comparison to children and younger adults. As this region has been linked to the generation and selection of new representations and the ability to organize multiple pieces of information, enhancing memory for item associations, it is difficult to find an argument why this region should be less involved in separating between remembered and omitted items in older adults in comparison to children and younger adults. It might be that the difference between remembered and not-remembered items in older adults does not reflect inadequate encoding, but rather poor consolidation or retrieval failures (see discussion above). Future research is needed to investigate the specific roles of different prefrontal brain regions in successful memory encoding across the lifespan, including a more fine-grained assessment of strategy use, and an examination of training-induced changes in functional connectivity.

Finally, in this study, we included confidence/imagery success ratings during encoding. Unfortunately, it was not possible to completely separate between brain activity associated with word pair encoding and brain activity associated with JOL's or imagery success ratings, due to the absence of jitter between the two types of events. In addition, it also was not possible to analyze data as a function of confidence ratings in this study, due to the low number of trials in each of the three confidence categories. Future work should seek to overcome these limitations so that the neural mechanisms associated with age group differences in training-induced changes in judgment of learning can be examined.

\section{Conclusions}

This is the first age-comparative fMRI study investigating traininginduced changes in SM effects during encoding, which refer to greater regional activity for associative information that is learned and retrieved successfully than information that is not remembered. This is also the first study investigating the impact of strategy instruction and practice on the SM effect. Encoding success is much less dependent on general age-related performance differences in the task in question, because the contrast compares remembered and not remembered items. We show similar SM effects across age groups before training, and that instruction and practice in a simple and effective strategy lead to similar changes in successful memory formation across the lifespan. These patterns extend the brain maintenance hypothesis (Lindenberger, 2014; Nyberg et al., 2012) to the within-person level. The reductions of the SM effect reflected greater activation increases for not remembered compared to remembered items, indicating that all age groups applied the trained strategy more systematically after instruction and practice. Importantly, these findings are obtained using age-matched controls, what has not been done in related intervention studies (e.g., Jones et al., 2006; Kirchhoff et al., 2012; Kondo et al., 2005; Nyberg et al., 2003); the effects are thus intervention-specific. Training-induced reductions in the SM effect were primarily observed for left frontal (inferior and dorsal prefrontal) regions, which is sensible, given that our intervention focuses on the flexible and systematic use of an effective encoding strategy.

\section{Acknowledgments}

This research was supported by grants from the German Research Foundation (grant number DFG SH550/2-1) to Yee Lee Shing and Yvonne Brehmer, the Swedish Research Council, the Swedish Research Council for Health, Working Life, and Welfare, Swedish Brain Power, an Alexander von Humboldt Research Award, and a donation from the af Jochnick Foundation to Lars Bäckman, and by grants from the Max Planck Society, the Federal Ministry of Education and Research, and a Gottfried Wilhelm Leibniz Award 2010 of the German Research Foundation (DFG) to Ulman Lindenberger. The first author also received postdoctoral funding from ERA-AGE, Future Leaders of Ageing Research in Europe (FLARE)/Swedish Council of Working Life and Social Research.

\section{References}

Avants, B.B., Epstein, C.L., Grossman, M., Gee, J.C., 2008. Symmetric diffomorphic image registration with cross-correlation: evaluating automated labeling of elderly and neurodegenerative brain. Med. Image Anal. 12, 26-41.

Avants, B.B., Tustison, N.J., Song, G., Cook, P.A., Klein, A., Gee, J.C., 2011. A reproducible evaluation of ANTs similarity metric performance in brain image registration. Neurolmage 54, 2033-2044.

Ball, K., Berch, D.B., Helmers, K.F., 2002. Effects of cognitive training interventions with older adults: a randomized controlled trial. J. Am. Med. Assoc. 288, 2271-2281.

Bherer, L., Kramer, A.F., Peterson, M.S., Colcombe, S., Erickson, K., Becic, E., 2006. Testing the limits of cognitive plasticity in older adults: application to attentional control. Acta Psychol. 123, 261-278.

Blumenfeld, R.S., Ranganath, C., 2007. Prefrontal cortex and long-term memory encoding: an integrative review of findings from neuropsychology and neuroimaging. Neuroscientist 13, 280-291.

Brehmer, Y., Stoll, G., Bergner, S., Benoit, R., von Oertzen, T., Lindenberger, U., 2004. Selection of unambiguous visual words, appropriate for children in age-comparable memory experiments: Results of a pilot study. Saarland University, Saarbrücken.

Brehmer, Y., Li, S.C., Müller, V., von Oertzen, T., Lindenberger, U., 2007. Memory plasticity across the life span: Uncovering children's latent potential. Dev. Psychol. 43, 465-478.

Cohn, M., Emrich, S.M., Moscovitch, M., 2008. Age-related deficits in associative memory: the influence of impaired strategic retrieval. Psychol. Aging 23, 93-103.

Derwinger, A., Stigsdotter Neely, A., Persson, M., Hill, R.D., Bäckman, L., 2003. Remembering numbers in old age: mnemonic training versus self-generated strategy training. Aging Neuropsychol. Cognit. 10, 202-214.

Dunlosky, J., Hertzog, C., 1998. Aging and deficits in associative memory: what is the role of strategy production? Psychol. Aging 13, 597-607. 
Fandakova, Y., Lindenberger, U., Shing, Y., 2015. Maintenance of youth-like processing protects against false memory in later adulthood. Neurobiol. Aging 36, 933-941.

Folstein, M.F., Folstein, S.E., McHugh, P.R., 1975. "Mini-mental state". A practical method for grading the cognitive state of patients for the clinician. J. Psychiatr. Res. 12, 189-198.

Habib, R., Nyberg, L., Nilsson, L.G., 2007. Cognitive and non-cognitive factors contributing to the longitudinal identification of successful older adults in the Betula study. Aging Neuropsychol. Cognit. 14, 257-273.

Hasselhorn, M., Jaspers, A., Hernando, M.-D., 1990. Typizitätsnormen zu zehn Kategorien für Kinder von der Vorschule bis zur vierten Grundschulklasse. Sprache Kognit. 9, 02-108.

Hertzog, C., Fulton, E.K., Mandviwala, L., Dunlosky, J., 2013. Older adults show deficits in retrieving and decoding associative mediators generated at study. Dev. Psychol. 49, 1127-1131.

Jenkinson, M., Bannister, P., Brady, M., Smith, S., 2002. Improved optimization for the robust and accurate linear registration and motion correction of brain images. Neurolmage 17 , $825-841$.

Jones, S., Nyberg, L., Sandblom, J., Stigsdotter Neely, A., Ingvar, M., Magnus Petersson, K., Bäckman, L., 2006. Cognitive and neural plasticity in aging: general and taskspecific limitations. Neurosci. Biobehav. Rev. 30, 864-871.

Kausler, D.H., 1994. Learning and Memory in Normal Aging. Academic Press, New York.

Kim, H., 2011. Neural activity that predicts subsequent memory and forgetting: a metaanalysis of $74 \mathrm{fMRI}$ studies. NeuroImage 54, 2446-2461.

Kirchhoff, B.A., Anderson, B.A., Barch, D.M., Jacoby, L.L., 2012. Cognitive and neural effects of semantic encoding strategy training in older adults. Cereb. Cortex 22, 788-799.

Kliegl, R., Baltes, P.B., 1987. Theory-guided analysis of mechanisms of development and aging mechanisms through testing-the-limits and research on expertise. In: Schooler, C., Schaie, K.W. (Eds.), Cognitive functioning and social structure over the life course. Ablex, Norwood, NJ, pp. 95-119.

Kliegl, R., Smith, J., Baltes, P.B., 1990. On the locus and process of magnification of age differences during mnemonic training. Dev. Psychol. 26, 894-904.

Kondo, Y., Suzuki, Y., Mugikura, S., Abe, N., Takahashi, S., Iijima, T., Fujii, T., 2005. Changes in brain activation associated with use of a memory strategy: a functional MRI study. Neurolmage 24, 1154-1163.

Kriegeskorte, N., Simmons, W.K., Bellgowan, P.S., Baker, C.I., 2009. Circular analysis in systems neuroscience: the dangers of double dipping. Nat. Neurosci. 12, 535-540.

Li, J., Morcom, A.M., Rugg, M.D., 2004. The effects of age on the neural correlates of successful episodic retrieval: an ERP study. Cogn. Affect. Behav. Neurosci. 4, 279-293.

Lindenberger, U., 2014. Human cognitive aging: Corriger la fortune? Science 346 $572-578$.

Maillet, D., Rajah, M.N., 2014. Age-related differences in brain activity in the subsequent memory paradigm: a meta-analysis. Neurosci. Biobehav. Rev. 45, 246-257.

Maldjian, J.A., Laurienti, P.J., Kraft, R.A., Burdette, J.H., 2003. An automated method for neuroanatomic and cytoarchitectonic atlas-based interrogation of fMRI data sets. Neurolmage 19, 1233-1239.

Maldjian, J.A., Laurienti, P.J., Burdette, J.H., 2004. Precentral gyrus discrepancy in electronic versions of the Talairach atlas. NeuroImage 21, 450-455.

Mark, R.E., Rugg, M.D., 1998. Age effects on brain activity associated with episodic memory retrieval. An electrophysiological study. Brain 121, 861-873.

Murphy, K., Garavan, H., 2005. Deriving the optimal number of events for an eventrelated fMRI study based on the spatial extent of activation. Neurolmage 27, 771-777.
Nyberg, L., Sandblom, J., Jones, S., Neely, A.S., Petersson, K.M., Ingvar, M., Bäckman, L., 2003. Neural correlates of training-related memory improvement in adulthood and aging. Proc. Natl. Acad. Sci. U. S. A. 100, 13728-13733.

Nyberg, L., Lövden, M., Riklund, K., Lindenberger, U., Bäckman, L., 2012. Memory aging and brain maintenance. Trends Cogn. Sci. 16, 292-305.

Paivio, A., 1969. Mental imagery in associative learning and memory. Psychol. Rev. 76, 241-263.

Park, H., Leal, F., Spann, C., Abellanoza, C., 2013. The effect of object processing in contentdependent source memory. BMC Neurosci. 14, 71.

Pudas, S., Persson, J., Josefsson, M., de Luna, X., Nilsson, L.-G., Nyberg, L., 2013. Brain characteristics of individuals resisting age-related cognitive decline over two decades. J. Neurosci. 33, 8668-8677.

Radloff, L.S., 1977. The CES-D Scale: a self-report depression scale for research in the general population. Appl. Psychol. Meas. 1, 385-401.

Rönnlund, M., Nyberg L, Bäckman, L, Nilsson, LG., 2005. Stability, growth, and decline in adult life span development of declarative memory: cross-sectional and longitudinal data from a population-based study. Psychol. Aging 20, 3-18.

Sanchez, C.E., Richards, J.E., Almli, C.R., 2012. Age-specific MRI templates for pediatric neuroimaging. Dev. Neuropsychol. 37, 379-399.

Scheithe, K., Bäuml, K.-H., 1995. Deutschsprachige Normen für Vertreter von 48 Kategorien. Sprache Kognit. 14, 39-43.

Schneider, W., Pressley, M., 1997. Memory development between two and twenty. Lawrence Erlbaum Associates, Mahwah, NJ.

Shing, Y.L., Werkle-Bergner, M., Li, S.C., Lindenberger, U., 2008. Associative and strategic components of episodic memory: a life-span dissociation. J. Exp. Psychol. Gen. 137, 495-513.

Singer, T., Lindenberger, U., Baltes, P.B., 2003. Plasticity of memory for new learning in very old age: a story of major loss? Psychol. Aging 18, 306-317.

Slotnick, S.D., 2003. Model fitting in $(\mathrm{n}+1)$ dimensions. Behav. Res. Methods 35 , $322-324$.

Smith, S.M., 2002. Fast robust automated brain extraction. Hum. Brain Mapp. 17, 143-155.

Smith, S.M., Jenkinson, M., Woolrich, M.W., Beckmann, C.F., Behrens, T.E., Johansen-Berg, H., ... Matthews, P.M., 2004. Advances in functional and structural MR image analysis and implementation as FSL. Neurolmage 23 (Suppl. 1), S208-S219.

Spaniol, J. Davidson, P.S.R, Kim, A.S.N., Han, H. Moscovitch, M. Grady, C.L 2009. Eventrelated fMRI studies of episodic encoding and retrieval: Meta-analyses using activation likelihood estimation. Neuropsychologia 47, 1765-1779.

Stark, C.E., Squire, L.R., 2001. When zero is not zero: The problem of ambiguous baseline conditions in fMRI. Proc. Natl. Acad. Sci. U. S. A. 98, 12760-12766.

Tulving, E., 1972. Episodic and semantic memory. In: Tulving, E., Donaldson, W. (Eds.), Organization of memory. Academic Press, New York, pp. 381-403.

Wang, T.H., Johnson, J.D., de Castelaine, M., Donley, B.E., Rugg, M.D., 2015. The effects of age on the neural correlates of recollection success, recollection-related cortical reinstatement, and post-retrieval monitoring. Cereb. Cortex 1-17.

Wechsler, D., 1981. Wechsler Adult Intelligence Scale-Revised. Psychological Corporation, New York.

Woolrich, M.W., Behrens, T.E., Beckmann, C.F., Jenkinson, M., Smith, S.M., 2004. Multilevel linear modelling for FMRI group analysis using Bayesian inference. Neurolmage 21, 1732-1747. 